\title{
Trajectory Coordinate Constraints in Multibody Railroad Vehicle Systems*
}

\author{
Hiroyuki SUGIYAMA $^{* *}$ and Ahmed A. SHABANA ${ }^{* * *}$ \\ ** Center for Collaborative Research, University of Tokyo \\ 4-6-1 Komaba, Meguro-Ku,Tokyo 153-8505, Japan \\ E-mail: hsugiy1@iis.u-tokyo.ac.jp \\ *** Department of Mechanical Engineering, University of Illinois at Chicago \\ 842 West Taylor, Chicago, Illinois 60607, USA
}

\begin{abstract}
In this investigation, the formulation of trajectory coordinate constraints in terms of the absolute coordinates is developed for the use in the computer simulations of railroad vehicle system applications. Examples of trajectory coordinate constraints are the specified forward velocity of a vehicle or a wheelset along a curved track or specified yaw angle of a vehicle or a wheelset with respect to a body trajectory coordinate system. The relationship between the trajectory coordinates and the absolute coordinates is defined and then used to write the trajectory constraints in terms of the absolute coordinates at the position, velocity, and acceleration levels. The motion of the trajectory coordinate system can be uniquely defined in the track body coordinate system using the arc-length coordinate defined along the space curve. It is demonstrated that the use of the trajectory coordinates leads to simple linear constraint equations, while the trajectory constraint equations become highly nonlinear functions of the absolute coordinates. This is mainly due to the nature of the nonlinear relationship between the absolute Cartesian and trajectory coordinates. A numerical example is presented in order to demonstrate the use of the proposed formulations in the analysis of multibody railroad vehicle systems.
\end{abstract}

Key words: Railroad Vehicle Dynamics, Multibody Dynamics, Trajectory Coordinate Constraints, Trajectory Coordinates, Absolute Coordinates.

\section{Introduction}

In the computer simulations of railroad vehicle systems ${ }^{(1)}$, it is often required to impose kinematic constraints on the motion trajectories ${ }^{(2-3)}$. Examples of these constraints are the specified forward velocity of a vehicle or a wheelset along a curved track or specified yaw angle of a vehicle or a wheelset with respect to a track coordinate system. It is also common to use absolute Cartesian coordinates and Euler angles or Euler parameters in general purpose multibody algorithms for the sake of generality and also to obtain a dynamic formulation that has a sparse matrix structure. This motion description leads to systematic and straightforward implementation of the wheel/rail contact formulations in existing general purpose multibody system computer algorithms ${ }^{(4-5)}$. This description also allows for exploiting advanced and well developed multibody system dynamics capabilities such as body flexibility. In this paper, the formulation of the trajectory constraints in terms of the absolute coordinates is developed for use in general purpose multibody computer algorithms. To this end, a set of trajectory coordinates, in terms of which trajectory constraints are linear and simple, is first introduced. The relationship between the trajectory coordinates and the absolute coordinates is then defined and used to write the trajectory 
constraints in terms of the absolute coordinates.

The paper that discusses the development of the trajectory coordinate constraints is organized as follows: In Section 2, the trajectory coordinates are introduced in order to explain general three-dimensional motion description of a rigid body in railroad vehicle applications, and the relationship between the trajectory and absolute coordinates is developed. In Section 3, using such relationships, the trajectory coordinate constraints at the position, velocity, and acceleration levels are expressed in terms of the absolute coordinates. The track space curve that enters into the trajectory constraint formulation is discussed in Section 4, while in Section 5 a numerical example is presented in order to demonstrate the use of the formulations developed in this investigation. Summary and conclusions drawn from this study are presented in Section 6 .

\section{Trajectory and Absolute Coordinates}

\subsection{Trajectory Coordinate System}

As shown in Fig. 1, the distance traveled by a body $i$ is defined by the arc-length $s^{i}$ measured along a track space curve. Given the geometry of the space curve and the parameter $s^{i}$, the location of the origin of a trajectory coordinate system $X^{t i} Y^{t i} Z^{t i}$ that follows the motion of body $i$ can be uniquely defined by the vector $\mathbf{R}^{t i}=\mathbf{R}^{t i}\left(s^{i}\right)$ as shown in Fig. 1, while the orientation of the trajectory coordinate system at this location with respect to the track coordinate system can be defined using the three Euler angles $\psi^{t i}, \theta^{t i}$, and $\phi^{t i}$ about the three axes $Z^{t i},-Y^{t i}$ and $-X^{t i}$, respectively. These three Euler angles can be uniquely defined in terms of the arc-length $s^{i}$ and their sequence is selected to be consistent with what is used by railroad industry. The transformation matrix, expressed in terms of these Euler angles, that defines the orientation of the trajectory coordinate system can be written as follows:

$$
\mathbf{A}^{t i}=\left[\begin{array}{lll}
\mathbf{i}^{t i} & \mathbf{j}^{t i} & \mathbf{k}^{t i}
\end{array}\right]
$$

where

$$
\begin{aligned}
& \mathbf{i}^{\mathrm{ti}}=\left[\begin{array}{llll}
\mathrm{c} \psi \mathrm{c} \theta & \mathrm{s} \psi \mathrm{c} \theta & \mathrm{s} \theta
\end{array}\right]^{T} \\
& \mathbf{j}^{\mathrm{ti}}=\left[\begin{array}{llll}
-\mathrm{s} \psi \mathrm{c} \phi+\mathrm{c} \psi \mathrm{s} \theta \mathrm{s} \phi & \mathrm{c} \psi \mathrm{c} \phi+\mathrm{s} \psi \mathrm{s} \theta \mathrm{s} \phi & -\mathrm{c} \theta \mathrm{s} \phi
\end{array}\right]^{T} \\
& \mathbf{k}^{t i}=\left[\begin{array}{lll}
-\mathrm{s} \psi \mathrm{s} \phi-\mathrm{c} \psi \mathrm{s} \theta \mathrm{c} \phi & \mathrm{c} \psi \mathrm{s} \phi-\mathrm{s} \psi \mathrm{s} \theta \mathrm{c} \phi & \mathrm{c} \theta \mathrm{c} \phi
\end{array}\right]^{T}
\end{aligned}
$$

In the preceding equation, $\mathrm{c}$ and $\mathrm{s}$ denote, respectively, $\cos$ and $\sin$. Note that if the space curve geometry is specified, one has $\psi^{t i}=\psi^{t i}\left(s^{i}\right), \theta^{t i}=\theta^{t i}\left(s^{i}\right)$, and $\phi^{t i}=\phi^{t i}\left(s^{i}\right)$. Since the trajectory coordinate system follows the body motion, its velocity and acceleration depend on the forward velocity and acceleration of the body.

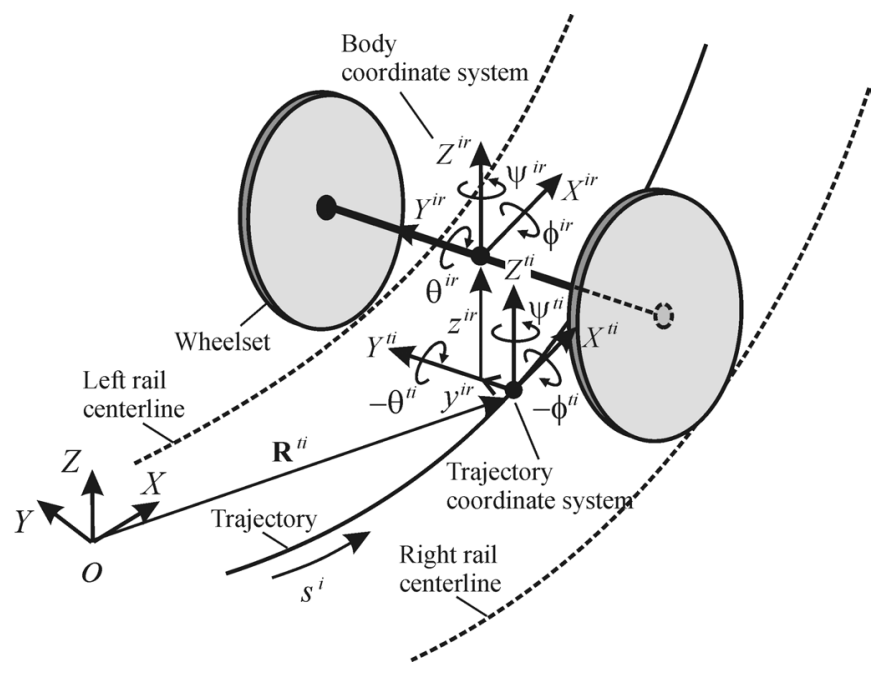

Fig. 1 Trajectory coordinates 
Using the Euler angle description, the angular velocity vector of the trajectory coordinate system defined in the global coordinate system is written in terms of the time derivatives of Euler angles as

$$
\boldsymbol{\omega}^{t i}=\mathbf{G}^{t i} \dot{\boldsymbol{\theta}}^{t i}=\left(\mathbf{G}^{t i} \frac{\partial \boldsymbol{\theta}^{t i}}{\partial s^{i}}\right) \dot{s}^{i}
$$

where the matrix $\mathbf{G}^{t i}$ is given as

$$
\mathbf{G}^{t i}=\left[\begin{array}{ccc}
0 & \sin \psi^{t i} & -\cos \psi^{t i} \cos \theta^{t i} \\
0 & -\cos \psi^{t i} & -\sin \psi^{t i} \cos \theta^{t i} \\
1 & 0 & -\sin \theta^{t i}
\end{array}\right]
$$

and $\boldsymbol{\theta}^{i t}=\left[\begin{array}{lll}\psi^{t i} & \theta^{t i} & \phi^{t i}\end{array}\right]^{T}$ are function of the arc-length coordinate $s^{i}$.

\subsection{Body Coordinate System}

Using the trajectory coordinate system whose location and orientation can be uniquely defined by the arc-length coordinate $s^{i}$, the global position vector of the center of mass of body $i$ can be written as

$$
\mathbf{R}^{i}=\mathbf{R}^{t i}+\mathbf{A}^{t i} \overline{\mathbf{u}}^{i r}
$$

where $\overline{\mathbf{u}}^{i r}$ is the position vector of the center of mass defined with respect to the trajectory coordinate system. This vector is defined as

$$
\overline{\mathbf{u}}^{i r}=\left[\begin{array}{lll}
0 & y^{i r} & z^{i r}
\end{array}\right]^{T}
$$

where $y^{i r}$ and $z^{i r}$ are, respectively, the center of mass coordinates in the lateral direction and in a direction normal to the plane that contains the track space curve. These coordinates are defined with respect to the trajectory coordinate system as shown in Fig. 1. The location of the center of mass in the longitudinal direction is assumed to be uniquely defined using the arc-length $s^{i}$, and for this reason, the first element of the vector $\overline{\mathbf{u}}^{i r}$ is selected to be zero.

In order to define the configuration of the body in the global system, a rotation matrix $\mathbf{A}^{i r}$ is introduced. This matrix defines the orientation of the centroidal body coordinate system $X^{i r} Y^{i r} Z^{i r}$ with respect to the trajectory coordinate system using the three Euler angles $\psi^{i r}, \phi^{i r}$, and $\theta^{i r}$. These three Euler angles are defined as rotations about the $Z^{i i}$, $X^{t i}$ and $Y^{t i}$ axes of the trajectory coordinate system. This sequence of Euler angles leads to the following transformation matrix that is used to define the orientation of the body coordinate system relative to the trajectory coordinate system:

$$
\mathbf{A}^{i r}=\left[\begin{array}{lll}
\mathbf{i}^{i r} & \mathbf{j}^{i r} & \mathbf{k}^{i r}
\end{array}\right]
$$

where

$$
\begin{aligned}
& \mathbf{i}^{i r}=\left[\begin{array}{lll}
\mathrm{c} \psi \mathrm{c} \theta-\mathrm{s} \psi \mathrm{s} \phi \mathrm{s} \theta & \mathrm{s} \psi \mathrm{c} \theta+\mathrm{c} \psi \mathrm{s} \phi \mathrm{s} \theta & -\mathrm{c} \phi \mathrm{s} \theta
\end{array}\right]^{T} \\
& \mathbf{j}^{i r}=\left[\begin{array}{llll}
-\mathrm{s} \psi \mathrm{c} \phi & \mathrm{c} \psi \mathrm{c} \phi & \mathrm{s} \phi
\end{array}\right]^{T} \\
& \mathbf{k}^{i r}=\left[\begin{array}{llll}
\mathrm{c} \psi \mathrm{s} \theta+\mathrm{s} \psi \mathrm{s} \phi \mathrm{c} \theta & \mathrm{s} \psi \mathrm{s} \theta-\mathrm{c} \psi \mathrm{s} \phi \mathrm{c} \theta & \mathrm{c} \phi \mathrm{c} \theta
\end{array}\right]^{T}
\end{aligned}
$$

Using the sequence of Euler angles for $\psi^{i r}, \phi^{i r}$, and $\theta^{i r}$, one can show that the angular velocity of the body coordinate system with respect to the trajectory coordinate system defined in the global coordinate system can be written in terms of the time derivatives of the orientation parameters as

$$
\boldsymbol{\omega}^{i r}=\mathbf{A}^{t i} \mathbf{G}^{i r} \dot{\boldsymbol{\theta}}^{i r}
$$

where $\boldsymbol{\theta}^{i r}=\left[\begin{array}{lll}\psi^{i r} & \phi^{i r} & \theta^{i r}\end{array}\right]^{T}$ and the matrix $\mathbf{G}^{i r}$ is given as

$$
\mathbf{G}^{i r}=\left[\begin{array}{ccc}
0 & \cos \psi^{i r} & -\sin \psi^{i r} \cos \phi^{i r} \\
0 & \sin \psi^{i r} & \cos \psi^{i r} \cos \phi^{i r} \\
1 & 0 & \sin \phi^{i r}
\end{array}\right]
$$


Clearly, the motion of body $i$ with respect to the trajectory coordinate system can be described using the translations $y^{i r}$ and $z^{i r}$ and the three relative rotations $\psi^{i r}, \phi^{i r}$, and $\theta^{i r}$. On the other hand, the motion of the trajectory coordinate system can be uniquely defined in the global coordinate system using the arc-length coordinate $s^{i}$. Therefore, total of six co-ordinates are required in order to uniquely define the general three-dimensional motion of the body in the global coordinate system. These six trajectory coordinates are given by

$$
\mathbf{p}^{i}=\left[\begin{array}{llllll}
s^{i} & y^{i r} & z^{i r} & \psi^{i r} & \phi^{i r} & \theta^{i r}
\end{array}\right]^{T}
$$

Using these trajectory coordinates, the global position vector of an arbitrary point on the body can be written as follows:

$$
\mathbf{r}^{i}=\mathbf{R}^{i}+\mathbf{A}^{i} \overline{\mathbf{u}}^{i}
$$

where $\mathbf{R}^{i}$ is the position vector of the center of mass of body $i$ defined by Eq.(5) and $\mathbf{A}^{i}$ is the rotation matrix that defines the orientation of the body coordinate system with respect to the global coordinate system. This matrix is defined by the following successive rotations:

$$
\mathbf{A}^{i}=\mathbf{A}^{t i} \mathbf{A}^{i r}
$$

The vector $\overline{\mathbf{u}}^{i}$ in Eq.(12) is the position vector of the arbitrary point on the body defined in the body coordinate system. Therefore, Eq.(12) can also be written using Eqs.(5) and (13) as

$$
\mathbf{r}^{i}=\mathbf{R}^{t i}+\mathbf{A}^{t i} \overline{\mathbf{u}}^{i r}+\mathbf{A}^{t i} \mathbf{A}^{i r} \overline{\mathbf{u}}^{i}
$$

This equation is expressed in terms of the six generalized trajectory coordinates defined by Eq.(11) since $\mathbf{R}^{t i}=\mathbf{R}^{t i}\left(s^{i}\right), \quad \mathbf{A}^{t i}=\mathbf{A}^{t i}\left(s^{i}\right), \overline{\mathbf{u}}^{i r}=\overline{\mathbf{u}}^{i r}\left(y^{i r}, z^{i r}\right)$, and $\mathbf{A}^{i r}=\mathbf{A}^{i r}\left(\psi^{i r}, \phi^{i r}, \theta^{i r}\right)$.

\subsection{Generalized Absolute Coordinates}

It can be seen from the expression of the general displacement given by Eq.(14) that the use of the trajectory coordinates can lead to a complex expression for the equations of motion of the rigid body as compared to the simple Newton-Euler equations. The use of these coordinates can also lead to the loss of the sparse matrix structure of the dynamic equations, and makes exploiting advanced flexible body capabilities less straightforward. For this reason, the following absolute Cartesian and orientation coordinates are commonly used as generalized coordinates in multibody computer algorithms:

$$
\mathbf{q}^{i}=\left[\begin{array}{ll}
\mathbf{R}^{i^{T}} & \boldsymbol{\theta}^{i^{T}}
\end{array}\right]^{T}
$$

where $\mathbf{R}^{i}$ is the global position vector of the origin of the body coordinate system and $\boldsymbol{\theta}^{i}$ is the rotational coordinates used to describe the orientation of the body coordinate system with respect to the global coordinate system. Since both the trajectory and absolute coordinates uniquely define the body configuration, the following relationship between the two sets of coordinates can be developed:

$$
\mathbf{p}^{i}=\mathbf{p}^{i}\left(\mathbf{q}^{i}\right)
$$

This relationship, which is highly nonlinear, will be used in the following section to write the trajectory constraints in terms of the generalized absolute coordinates.

\section{Trajectory Coordinate Constraints}

\subsection{Translational Coordinate Constraints}

The constraints on the translational trajectory coordinates $\mathbf{p}_{R}^{i}=\left[\begin{array}{lll}s^{i} & y^{i r} & z^{i r}\end{array}\right]^{T}$ are first considered. In this case, the trajectory coordinate constraints can be written as 


$$
C_{l}=p_{l}^{i}-f(t)=0, \quad l=1,2,3
$$

where $p_{l}^{i}$ denotes the $l$-th component of the trajectory coordinate vector $\mathbf{p}^{i}$ defined by Eq.(11) and $f(t)$ is an explicit function of time. It is important to note that the preceding equation is linear and simple when expressed in terms of the trajectory coordinates $p_{l}^{i}$. The constraint, however, can be highly nonlinear when written in terms of the absolute Cartesian coordinates. This is mainly due to the nature of the nonlinear relationship between the absolute and trajectory coordinates as given by Eq.(16). If a vehicle body is assumed to be driven by a specified forward velocity $V$ along the centerline of straight/curved track, the constant trajectory forward velocity constraint can be defined using Eq.(17) $(l=1)$ in terms of the trajectory coordinate $s^{i}$ as

$$
C=s^{i}-s_{0}^{i}-V t=0
$$

where $s_{0}^{i}$ is the initial arc-length coordinate. Using the preceding constraint equation, the origin of the trajectory coordinate system always follows a specified trajectory and moves with a constant velocity $V$. In an absolute coordinate formulation, the trajectory coordinates $\mathbf{p}_{R}^{i}=\left[\begin{array}{lll}s^{i} & y^{i r} & z^{i r}\end{array}\right]^{T} \quad$ must be expressed in terms of the absolute coordinates. Using Eq.(5), one has the following relationship between the two sets of translational coordinates:

$$
\mathbf{g}\left(s^{i}, y^{i r}, z^{i r}\right)=\mathbf{R}^{i}-\mathbf{R}^{t i}-\mathbf{A}^{t i} \overline{\mathbf{u}}^{i r}=\mathbf{0}
$$

This equation can be used to determine the trajectory coordinates $s^{i}, y^{i r}$, and $z^{i r}$ for a given translational Cartesian coordinates $\mathbf{R}^{i}=\left[\begin{array}{lll}R_{x}^{i} & R_{y}^{i} & R_{z}^{i}\end{array}\right]^{T}$. Note that since the preceding equation is nonlinear function of the trajectory coordinates $s^{i}, y^{i r}$, and $z^{i r}$, an iterative procedure such as Newton-Raphson method must be used in order to determine the three translational trajectory coordinates. Therefore, imposing the constraints at the position level in an absolute coordinate formulation requires the solution of a nonlinear system of algebraic equations.

Differentiating Eq.(19) with respect to time, the time derivatives of the trajectory coordinates $s^{i}, y^{i r}$, and $z^{i r}$ can be written in terms of the time derivatives of the Cartesian coordinates $\mathbf{R}^{i}$ as

$$
\dot{\mathbf{p}}_{R}^{i}=\boldsymbol{\Psi}^{i} \dot{\mathbf{R}}^{i}
$$

where $\dot{\mathbf{p}}_{R}^{i}=\left[\begin{array}{lll}\dot{s}^{i} & \dot{y}^{i r} & \dot{z}^{i r}\end{array}\right]^{T}$ and $\boldsymbol{\Psi}^{i}$ is the $3 \times 3$ velocity transformation matrix defined as

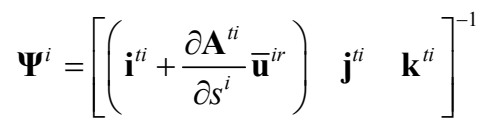

where the vector $\mathbf{i}^{t i}$ is a unit tangent to the space curve given by $\mathbf{i}^{t i}=\partial \mathbf{R}^{t i} / \partial s^{i}$ and can be determined from the track geometry data for a given $s^{i}$. Using the chain rule of differentiation, $\partial \mathbf{A}^{t i} / \partial s^{i}$ can be written as

$$
\frac{\partial \mathbf{A}^{t i}}{\partial s^{i}}=\frac{\partial \mathbf{A}^{t i}}{\partial \psi^{t i}} \frac{\partial \psi^{t i}}{\partial s^{i}}+\frac{\partial \mathbf{A}^{t i}}{\partial \theta^{t i}} \frac{\partial \theta^{t i}}{\partial s^{i}}+\frac{\partial \mathbf{A}^{t i}}{\partial \phi^{t i}} \frac{\partial \phi^{t i}}{\partial s^{i}}
$$

Differentiating Eq.(20) with respect to time, one obtains

$$
\ddot{\mathbf{p}}_{R}^{i}=\boldsymbol{\Psi}^{i} \ddot{\mathbf{R}}^{i}+\dot{\mathbf{\Psi}}^{i} \dot{\mathbf{R}}^{i}
$$

where $\dot{\mathbf{\Psi}}^{i} \dot{\mathbf{R}}^{i}$ includes all the terms that are quadratic in the generalized velocities of $\mathbf{R}^{i}$. Using Eq.(21), the Jacobian matrix of the constraints of the type given by Eq.(17) can be obtained. This Jacobian matrix associated with the Cartesian coordinates $\mathbf{R}^{i}$ is written for a constraint on a trajectory coordinate $p_{l}^{i}, l=1,2,3$, as 


$$
\frac{\partial C_{l}}{\partial \mathbf{R}^{i}}=\left[\begin{array}{lll}
\psi_{l 1}^{i} & \psi_{l 2}^{i} & \psi_{l 3}^{i}
\end{array}\right]
$$

where $\psi_{l m}^{i}$ refers to the $l$-th row and $m$-th column component of the matrix $\Psi^{i}$. Accordingly, the constraint equation at the acceleration level can be written as

$$
\sum_{m=1}^{3} \psi_{l m}^{i} \ddot{R}_{m}^{i}=\left(Q_{d}\right)_{l}
$$

where $\left(Q_{d}\right)_{l}$ is the quadratic velocity term given as

$$
\left(Q_{d}\right)_{l}=-\left(\dot{\Psi}^{i} \dot{\mathbf{R}}^{i}\right)_{l}+\frac{\partial^{2} f(t)}{\partial t^{2}}
$$

where $\left(\dot{\Psi}^{i} \dot{\mathbf{R}}^{i}\right)_{l}$ refers to the $l$-th component of the quadratic velocity vector $\dot{\boldsymbol{\Psi}}^{i} \dot{\mathbf{R}}^{i}$. This result clearly shows the complexity of the trajectory constraints at the acceleration level when the absolute Cartesian coordinates are used. Recall that the constraint Jacobian matrix becomes constant and the quadratic velocity vectors are always equal to zero when the trajectory coordinates are used as the generalized coordinates.

\subsection{Orientation Coordinate Constraints}

The trajectory coordinate constraint equations associated with the orientation of vehicle body $i$ can be written as follows:

$$
C_{l}=\theta_{l}^{i r}-\theta_{0 l}^{i r}=0, \quad l=1,2,3
$$

where $\theta_{l}^{i r}$ refers to the $l$-th component of the orientation trajectory coordinates $\boldsymbol{\theta}^{i r}=\left[\begin{array}{lll}\psi^{i r} & \phi^{i r} & \theta^{i r}\end{array}\right]^{T}$ and $\theta_{0 l}^{i r}$ is the value of this coordinate at the initial configuration. If it is required to constrain the yaw motion of a body with respect to the trajectory coordinate system, the following simple constraint equation can be defined using Eq.(27) $(l=1)$ in terms of the yaw angle $\psi^{i r}$ with respect to the trajectory coordinate system:

$$
C=\psi^{i r}-\psi_{0}^{i r}=0
$$

where $\psi_{0}^{i r}$ is assumed to be the initial yaw angle of the body with respect to the trajectory coordinate system and as a result of imposing the preceding constraint, the yaw angle of the body always remains the same as the initial yaw angle defined with respect to the trajectory coordinate system.

In order to define the preceding constraint equations in terms of the absolute coordiantes, the matrix that defines the orientation of the body coordinate system with respect to the trajectory coordinate system is expressed as follows:

$$
\mathbf{A}^{i r}=\mathbf{A}^{t^{T}{ }^{T}} \mathbf{A}^{i}
$$

Using this equation and assuming that the matrix $\mathbf{A}^{i}$ is known in terms of the absolute orientation coordinates, one can determine the Euler angles $\psi^{i r}, \phi^{i r}$, and $\theta^{i r}$ for the given absolute orientation coordinates $\boldsymbol{\theta}^{i}$ and the arc-length $s^{i}$ which is used to determine $\mathbf{A}^{t i}$. The arc-length $s^{i}$ can be determined for given values of the absolute generalized coordinates $\mathbf{R}^{i}$ as previously discussed in Eq.(19). The absolute angular velocity vector of the body with respect to the trajectory coordinate system can be written as

$$
\boldsymbol{\omega}^{i r}=\boldsymbol{\omega}^{i}-\boldsymbol{\omega}^{t i}
$$

where $\boldsymbol{\omega}^{i}$ is the angular velocity vector of body $i$ defined in the global coordinate system. The angular velocity vector $\boldsymbol{\omega}^{t i}$ of the trajectory coordinate system with respect to the global coordinate system is given by Eq.(3). Using Eq.(30), one has 


$$
\dot{\boldsymbol{\theta}}^{i r}=\left(\mathbf{A}^{t i} \mathbf{G}^{i r}\right)^{-1}\left(\mathbf{G}^{i} \dot{\boldsymbol{\theta}}^{i}-\mathbf{G}^{t i} \frac{\partial \boldsymbol{\theta}^{t i}}{\partial s^{i}} \dot{s}^{i}\right)
$$

where $\boldsymbol{\omega}^{i}=\mathbf{G}^{i} \dot{\boldsymbol{\theta}}^{i}$ and $\boldsymbol{\omega}^{i r}=\mathbf{A}^{t i} \mathbf{G}^{i r} \dot{\boldsymbol{\theta}}^{i r}$. Note that since the time derivative of the arc-length $s^{i}$ is given by the first equation of Eq.(20), the preceding equation can be re-written in terms of the time derivatives of the generalized absolute Cartesian coordinates as

$$
\dot{\boldsymbol{\theta}}^{i r}=\boldsymbol{\Phi}^{i} \dot{\mathbf{q}}^{i}
$$

where $\dot{\mathbf{q}}^{i}=\left[\begin{array}{ll}\dot{\mathbf{R}}^{i} & \dot{\boldsymbol{\theta}}^{i^{T}}\end{array}\right]^{T}$ and $\boldsymbol{\Phi}^{i}$ is a velocity transformation matrix defined as

$$
\boldsymbol{\Phi}^{i}=\left(\mathbf{A}^{t i} \mathbf{G}^{i r}\right)^{-1}\left[\begin{array}{llll}
-\mathbf{G}^{t i} \frac{\partial \boldsymbol{\theta}^{i i}}{\partial s^{i}} \psi_{11}^{i} & -\mathbf{G}^{t i} \frac{\partial \boldsymbol{\theta}^{t i}}{\partial s^{i}} \psi_{12}^{i} & -\mathbf{G}^{t i} \frac{\partial \boldsymbol{\theta}^{t i}}{\partial s^{i}} \psi_{13}^{i} & \mathbf{G}^{i}
\end{array}\right]
$$

Differentiating Eq.(32) with respect to time, one obtains

$$
\ddot{\boldsymbol{\theta}}^{i r}=\boldsymbol{\Phi}^{i} \ddot{\mathbf{q}}^{i}+\dot{\boldsymbol{\Phi}}^{i} \dot{\mathbf{q}}^{i}
$$

where $\dot{\boldsymbol{\Phi}}^{i} \dot{\mathbf{q}}^{i}$ includes all the terms that are quadratic in the generalized velocity of the absolute coordinates. Using Eq.(33), the constraint Jacobian matrix associated with the Cartesian coordinates can be written as

$$
\frac{\partial C_{l}}{\partial \mathbf{q}^{i}}=\left[\begin{array}{llll}
\Phi_{l 1}^{i} & \Phi_{l 2}^{i} & \ldots & \Phi_{l n_{q}^{i}}^{i}
\end{array}\right]
$$

where the index $l$ refers to the constrained rotation coordinate (see Eq.(27)), $\Phi_{l m}^{i}$ refers to the $l$-th row and $m$-th column component of the matrix $\boldsymbol{\Phi}^{i}$, and $n_{q}^{i}$ is the number of absolute Cartesian generalized coordinates of body $i ; n_{q}^{i}=7$ if four Euler parameters are used to describe the orientation of the body coordinate system in the absolute coordinate formulation. Accordingly, the constraint equation at the acceleration level can be written as

$$
\sum_{m=1}^{n_{q}^{i}} \Phi_{l m}^{i} \ddot{q}_{m}^{i}=\left(Q_{d}\right)_{l}
$$

where $\left(Q_{d}\right)_{l}$ is the quadratic velocity term given as

$$
\left(Q_{d}\right)_{l}=-\left(\dot{\boldsymbol{\Phi}}^{i} \dot{\mathbf{q}}^{i}\right)_{l}
$$

where $\left(\dot{\boldsymbol{\Phi}}^{i} \dot{\mathbf{q}}^{i}\right)_{l}$ refers to the $l$-th component of the vector $\dot{\boldsymbol{\Phi}}^{i} \dot{\mathbf{q}}^{i}$ in Eq.(34). The preceding equations clearly show the complexity of the trajectory constraints at the acceleration level when the absolute Cartesian coordinates are used.

\section{Track Space Curve}

Since the location and the orientation of the trajectory coordinate system are, respectively, defined by the arc-length coordinate $s^{i}$ as $\mathbf{R}^{t i}=\mathbf{R}^{t i}\left(s^{i}\right)$ and $\boldsymbol{\theta}^{t i}=\boldsymbol{\theta}^{t i}\left(s^{i}\right)$, the track space curve data enter into the constraint equations. For this reason, pre-processing steps are required in order to prepare the position, orientation, and the derivatives of the trajectory coordinate system for a given arc-length $s^{i}$. Since the tangent to the space curve is defined by the first derivative of the position vector $\mathbf{R}^{t i}$ as $\mathbf{i}^{i i}=\partial \mathbf{R}^{t i} / \partial s^{i}$, the derivatives of the position vector $\mathbf{R}^{t i}$ need to be calculated up to the third order for the use in the constraint equations at the position, velocity, and acceleration levels. That is, the second derivatives of the tangent that appears in the quadratic velocity vector of the trajectory coordinate constraint equations at the acceleration level lead to the third derivatives of the position vector $\mathbf{R}^{t i}$ as follows:

$$
\ddot{\mathbf{i}}^{t i}=\frac{\partial \mathbf{i}^{t i}}{\partial s^{i}} \ddot{s}^{i}+\frac{\partial^{2} \mathbf{i}^{i i}}{\partial s^{i 2}}\left(\dot{s}^{i}\right)^{2}=\frac{\partial^{2} \mathbf{R}^{t i}}{\partial s^{i 2}} \ddot{s}^{i}+\frac{\partial^{3} \mathbf{R}^{t i}}{\partial s^{i 3}}\left(\dot{s}^{i}\right)^{2}
$$


Accordingly, the following track space curve data needs to be prepared in advance:

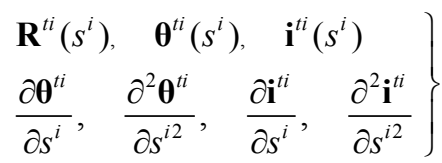

where $\partial \boldsymbol{\theta}^{t i} / \partial s^{i}$ is used to define $\partial \mathbf{A}^{t i} / \partial s^{i}$ in Eq.(22) and the absolute angular velocity vector defined by Eq.(31), while $\partial^{2} \boldsymbol{\theta}^{t i} / \partial s^{i 2}$ is used to define $\partial^{2} \mathbf{A}^{t i} / \partial s^{i 2}$ that appears in the constraint equations at the acceleration level. All these data are interpolated for a given arc-length coordinate $s^{i}$ that is determined by solving Eq.(19) at every time step.

\section{Numerical Example}

In order to demonstrate the use of the trajectory coordinate constraints developed in this investigation, a numerical example of a single wheelset as shown in Fig. 1 is considered. The wheelset mass used in this example is assumed to be $1568 \mathrm{~kg}$ and the moments of inertia are assumed to be $I_{y y}=168 \mathrm{kgm}^{2}, I_{x x}=I_{z z}=656 \mathrm{kgm}^{2}$. The wheels are profiled with approximate conicity of $1 / 40$, while the rail profile is assumed to be of the A.R.E.A. type of the Association of American Railroad. The track preprocessor discussed in the preceding section is used to generate the track space curve data that enter into the formulation of the trajectory coordinate constraints. The track is assumed to consist of $30 \mathrm{~m}$ tangent segment followed by $30 \mathrm{~m}$ spiral segment used to define a 1.5 degree constant curve segment as shown in Fig. 2. A $7.5 \mathrm{~mm}$ super-elevation is assumed on the right and left rails with respect to the center reference space curve. The longitudinal motion of the wheelset is constrained by imposing a constraint on the trajectory coordinate. Using this constraint, the wheelset is assumed to be driven by a constant forward velocity $V=5 \mathrm{~m} / \mathrm{s}$ along the tangent to the curved track centerline. The embedding contact constraint formulation is used to model the tread contact between the wheel and rail, while the elastic contact approach is employed to model the flange contact (see Ref.(4) for more details on the contact formulation). Figure 3 shows the arc-length coordinate as function of time. The result presented in this figure demonstrates that the wheelset is traveling along the curved track with the specified constant velocity of $5 \mathrm{~m} / \mathrm{s}$. Figure 4 shows the normal contact forces on the right wheel. It can be seen from this figure that the wheel flange comes in contact with the rail when the wheelset is in the spiral region. The lateral displacement of the wheelset with respect to the trajectory coordinate system is presented in Fig. 5. One can see from this figure that the wheelset starts moving laterally once it enters into the spiral region and the lateral displacement approaches a constant value when the wheelset has the flange contact and is in the constant curve region.

\section{Summary and Conclusions}

In this investigation, the formulation and implementation of the trajectory coordinate constraints are developed using the absolute coordinates that are commonly used in general purpose multibody algorithms for the sake of generality. To this end, the relationship between the trajectory coordinates and the absolute coordinates is developed and then used to write the trajectory constraints in terms of the absolute coordinates at the position, velocity, and acceleration levels. It is shown that these constraints are linear in the trajectory coordinates, while highly nonlinear in terms of the absolute coordinates. The formulation developed in this study is implemented in a general multibody computer algorithm. A numerical example is presented in order to demonstrate the use of the proposed formulations in the analysis of multibody railroad vehicle systems. 


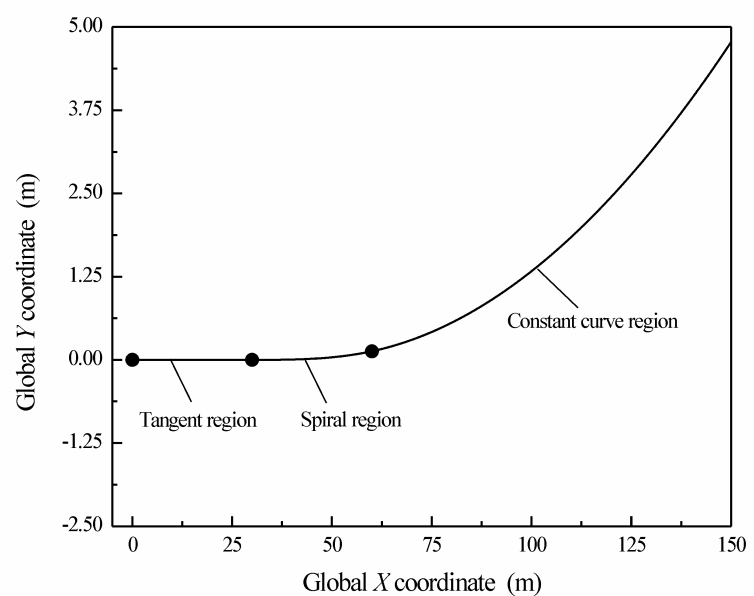

Fig. 2 Curved track

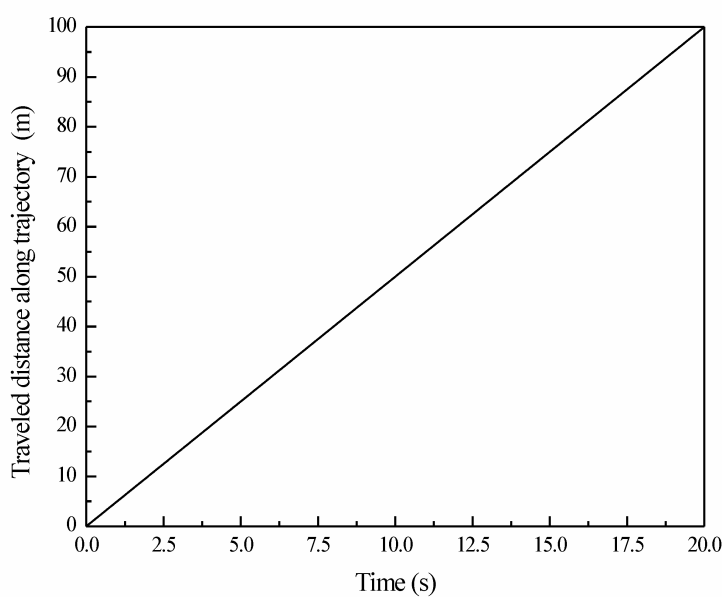

Fig. 3 Traveled distance of the wheelset along the trajectory

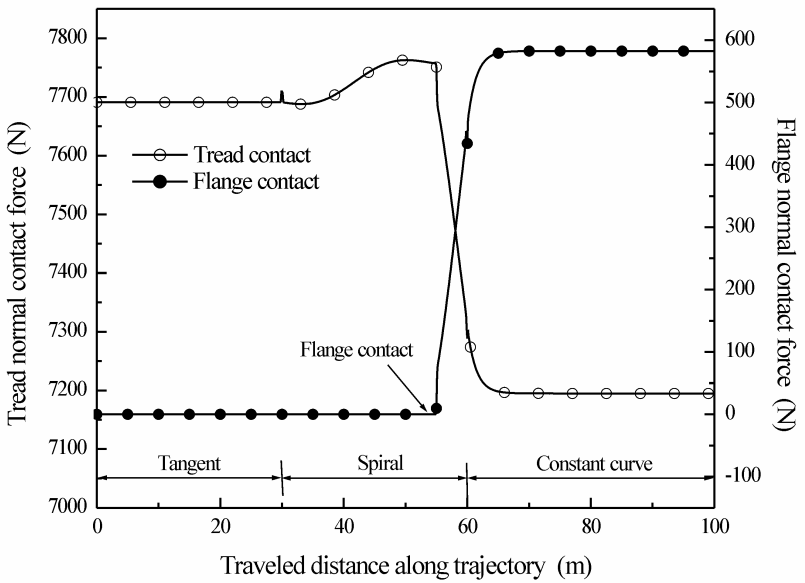

Fig. 4 Normal contact forces on the right wheel 


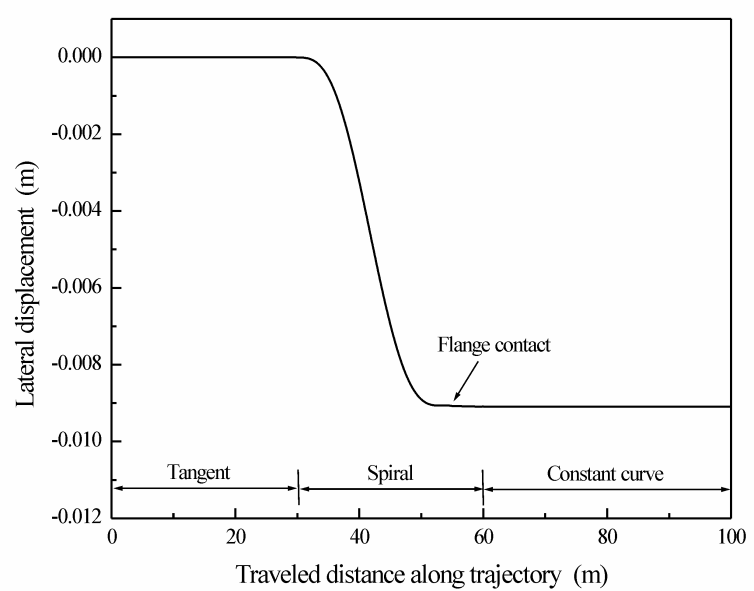

Fig. 5 Lateral displacement with respect to the trajectory coordinate system

\section{Acknowledgement}

This research was supported by the Federal Railroad Administration. This financial support is very much appreciated.

\section{References}

(1) Popp, K., and Schiehlen, W., 1993, Fahrzeugdynamik, Teubner, Stuttgart.

(2) Shabana, A. A., Zaazaa, E. K, Escalona, L. J, and Sany, J. R., 2004, Development of Elastic Force Model for Wheel/Rail Contact Problems, Journal of Sound and Vibration, vol. 269, pp. 295-325.

(3) SIMPACK Element Catalogue ver.8.7, 2004, INTEC.

(4) Shabana, A. A., Tobaa, M., Sugiyama, H. and Zaazaa, K., 2005, On the Computer Formulations of the Wheel/Rail Contact, Nonlinear Dynamics, vol. 40, pp. 169-193.

(5) Shabana, A. A., Zaazaa, K., and Sugiyama, H., 2007, Railroad Vehicle Dynamics: A Computational Approach, CRC: Taylor and Francis. 